International Symposium on Micromechanics:

Homogenization, Heterogenization, and Strength

DOE/ER/13687--8

The George Hermann 70th Anniversary Symposium

DE92 016852

\title{
Local-Equilibrium Formalism Applied to Mechanics of Solids
}

\author{
by \\ Joseph Kestin \\ Brown University \\ Providence, Rhode Island, U.S.A.
}

\section{DISCLAIMER}

This report was prepared as an account of work sponsored by an agency of the United States Neither the United Stales Government nor any agency thercof, nor any of their implied, or assumes any legal liability or responsi. ernployees, makes any warrang, express or isefulness of any information, apparatus, product, or bility for the accuracy, completeness, or usefulness of any inge privately owned rights. Referprocess disclosed, or represents that its use would not in or service by trade name, trademark, ence herein to any specific commercial product, prosess, or imply its endorsement, recominanufacturer, or otherwise does not necessarily constifuet or any agency thereof. The views mendation, or favoring by the United States Governmentarily state or reflect thuse of the

and opinions of authors expressed herein do not

March - April, 1991

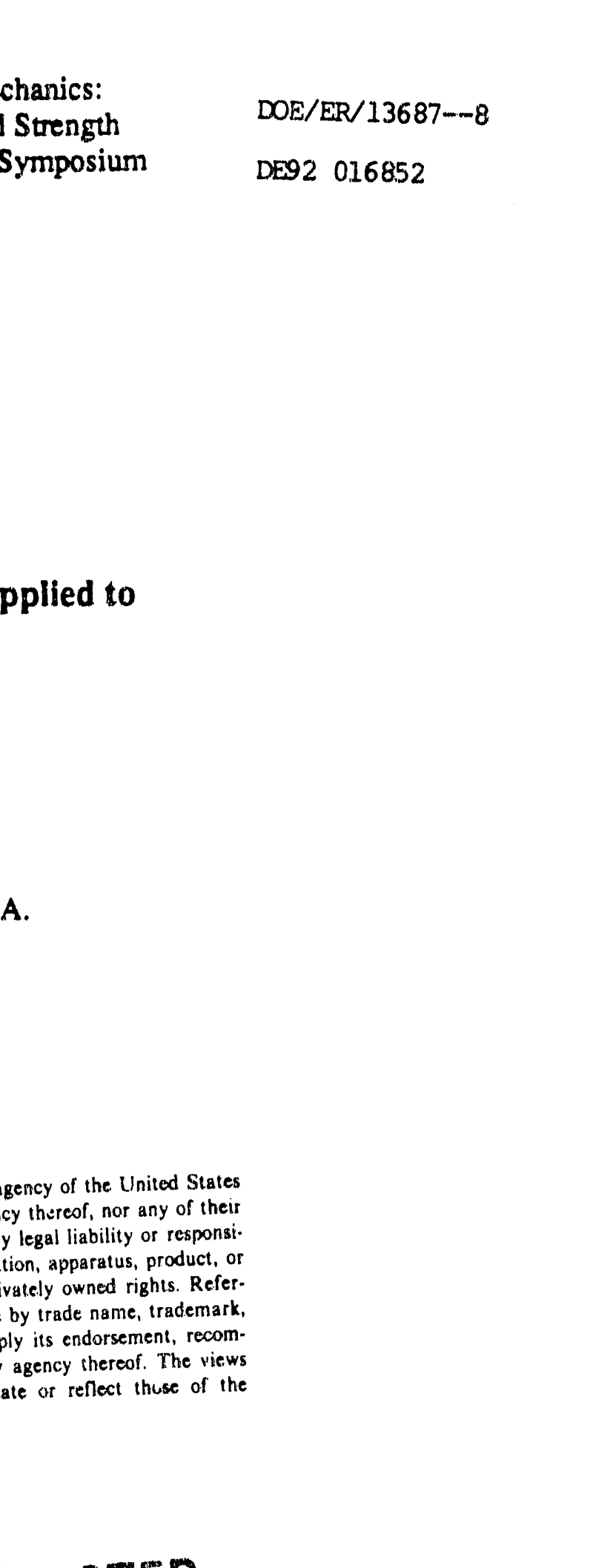




\section{Contents}

Denes

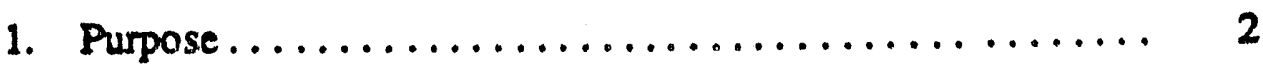

2. Background $\ldots \ldots \ldots \ldots \ldots \ldots \ldots \ldots \ldots \ldots \ldots$

3. Basics ............................ 5

4. State ......................... 6

5. Accompanying equilibrium state; accompanyirig reversible process $\ldots \ldots \ldots \ldots \ldots \ldots \ldots \ldots 8$

6. Entropy and thermodynamic temperature $\ldots \ldots \ldots \ldots \ldots . \ldots 10$

7. Hypothesis of local equilibrium

(principle of local state) $\ldots \ldots \ldots \ldots \ldots \ldots \ldots \ldots 11$

8. Entropy balance. Entropy production. .............. 13

Reierences $\ldots \ldots \ldots \ldots \ldots \ldots \ldots \ldots \ldots \ldots \ldots \ldots \ldots$

Appendix. The local-equilibrium formalism ............ 17 


\section{Local-Equilibrium Formalism Applied to}

Mechanics of Solids

by

Joseph Kestin

Brown University, Providence, Rhode Island, 01921, U.S.A.

Physics, whether we choose to call Thermodynamics should not be impotent in the face of any situation which can be completely characterized by measurements with macroscopic instruments.

P. W. Bridgman, 1950

Abstract

The lecture starts with an expression of good wishes to George Herrmann on the occasion of his seventieth birthday and continues with a lament that the majority of research workers in the field of solid mechanics have failed to appreciate the power and relevance of "conventional" thermodynamics which is based on the acceptance of the hypothesis of local equilibrium (principle of local state).

The lecture then proceeds to motivate the essential concepts of conventional thermodynamics and emphasizes the differences between the description of nonequilibrium states in physical space and equilibrium states in the Gibbsian phase space. It is asserted that the subject acquires its simplest form by the recognition of the relevance of Bridgman's intemal variables. With their aid it is possible to define the accompanying equilibrium state and the accompanying reversible process. An elimination of internal energy between the field equation of energy (First Law) and the Gibbs equation in rate form results in an explicit expression for the local rate of entropy production, $\theta$.

It is asserted that the preceding elements supplemented with appropriate rare equations result in a closed system of partial differential equations whose solution, subject to appropriate initial and boundary conditions, constitutes the process ("history") under consideration. 


\section{Bupose}

My purpose in preparing this, of necessity brief lecture, is twofold. First I wish to associate myself with all the Symposium participants and friends who could not be present with us, in wishing George Hermann many healthy years of productive work and undiminishing creativity.

My second purpose is once more to give an outline of the application of conventional thermodynamics to the analysis of problems in the mechanics of solids with emphasis on nonelastic behavior. Conventional thermodynamics rests on the hypothesis of local-equilibrium (which I prefer to call the principle of local state), made explicit independently by J. Meixner [1] and 1. Prigogine [2] and codified, in its application to the mechanics of fluids by $S$. R. de Groot and P. Mazur [3].

In doing s?, I express the conviction that a systematic and sustained development of its power in the study of stress and strain in metals would allow the subject to realize J. W. Gibbs's [4] philosophical credo:

One of the principal objects of theoretical research in any department of knowledge is to find the puint of view from which the subject appears in its greatest simplicity.

In the year 1979 [5] I wrote:

If I had been asked--say fifteen years ago \{i.e., circa 1965\}--whether there were any difficulties in applying the principles of thermodynamics to the inelastic processes which occur in strained metals, I would have, most emphatically, answered in the negative.

The intervening years reveajed two principal difficulties. The first principal difficulty resulted from a vigorous attack on the tenets of conventional thermodynamics. It is true that certain features of the local-equilibrium formalism suffer from aesthetic flaws. The formalism leads to infinite velocities of propagation of small disturbances and results in parabolic rather than 
hyperbolic differential equations for thermal conduction and diffusion. It evades the problem of assigning a numerical value of entropy and temperature to nonequilibrium states, which are always continuously present in irreversible processes, by equating them with those of an accompanying equilibrium state and introduces, without quite formally admitting it, an expression for the entropy flux which only approximates that derived in kinetic theory. However, this is no reason to reject ih because it is a fact that nearly all of applicable fluid mechanics and an impressive variety of phenomena treated in such consummate detail by J. Meixner and H. G. Reik [1], as well as S. R. de Groot and P. Mazur [2], rest firmly on the acceptance of the principle of local state." The concomitant Onsager-Casimir relations, just as countless other consequences and corollaries, have found reliable confirmation by experiment, as shown in detail, e.g., by D. G. Miller [6]. Taking a pragmatic point of view, I maintain that this simple formalism satisfies one more Gibbsian credo:

... it is the office of theoretical investigation to give the form in which

the results of experiments may be expressed.

In shor, I believe that a more widespread use of conventional thermodynamics in the study of problems in the mechanics of solids will lead to simplicity of presentation and agreement with experimental results.

The second difficulty, which I discovered subsequently, is that this marriage between thermodynamics and continuum mechanics was neither simple nor straightforward. The difficulties were (and still are) particularly acute in the study of plastic deformations, starting with P. W. Bridgman's $[7,8]$ belief that an equation of state for a plastically deformed bar "does not exist" and that its state is immersed in a "sea of irreversibility." As noted before [9], we may be baffled by superficial "paradoxes" and, in general, the problem of enumerating a proper set of

\footnotetext{
- There exists a similar situation in mechanics (icules proportions guardées). We have not scornfully forsaken Newtonian mechanics just because it is based on the nebulous concept of an inertial frame of reference, equales the $m$ in two equations without reference to an axiom and fails to calculate a correct orbit for the planet Mercury. Are we forbidden to calculate the orbit of balls on a billiard table wi:tyout reference to general relativity or, even, without accounting for Coriolis forces?
} 
independent and dependent thermodynamic variables has been solved for a limited number of cases only. This weakness still persists in relation to the essential internal variables to be introduced later.

\section{Backeround}

The subject of "conventional" thermodynamics, as it is taught, more or less correctly, in the academic departments of engineering in the civilized world, and the subject of solid mechanics, often taught as strength of materials, have developed largely independently of each other. Although both, ultimately, allow engineers to use them for design and testing with rather satisfactory results, they are not consistent with each other. They certainly failer. to converge to this day. In a situation like this it is quite natural to think that, perhaps, the foundations of both disciplines are at fault. It is interesting to note that thoughts of this lind compelled Bridgman, when he analyzed plastic deformation, to write:

There is a two-fold problem here--the problem of extending the conceptual machinery so as to be able to handle the new situations, and the experimental problem of finding what the facts are in the new domain.

It is symptomatic that several circles of scientists felt compelled to invent a new and more "rational" version of thermodynamics, pungently presented by C. Truesdell [10]. Nevertheless, I still believe that "nothing so drastic as a complete revision of thermodynamics is really needed" [5]. The development of "rational," and other competing versions of thermodynamics, have compelled the more traditional practitioners of the subject to sharpen their thoughts, concepts, and words. However, since the revisionists prefer to work in universes of discourse which differ from that of the traditional thermodynamicist's, there has arisen a new yocabulary, and the alternative

\footnotetext{
- Several oher versions have also been proposed. See Ref. [11].
} 
camps have bogged down in semantic difficulties. In short, in this first year of the last decade of the twentieth century, a student of the joint discipline of continuum thermodynamics and mechanics of solids faces a veritable Tower of Babel.

I do not intend to create a dictionary of the several languages now in use. And just like in linguistics, the words do not stand to each other in a one-to-one correspondence. I shall merely explain the meaning of several concepts, which are essential to my presentation, in order to let my audience understand the resulting formalism--whether they accept it as valid or not.

\section{Basics}

At the most fundamental level, thermodynamics speaks about systems rather than materials and makes a clear distinction between the state of the system (equilibrium or nonequilibrium) and the process (reversible or irreversible) undergone by the system. In this universe of discourse a material with time-dependent properties or a material with memory represent systems which perform a process during the time they are observed. Similarly, history represents a process which has occurred between a specified initial state and the state under consideration. Relations between the properties (variables) of equilibrium states are formulated as equations of state, a special role being reserved for fundamental equations of state from which all others follow by differentiation alone. The corresponding statements about nonequilibrium states are in need of an elaboration which will be presented in Sections 47.

A reversible process is conceived as a continuous sequence of equilibrium states in time whereas an irreversible process consists of a spatially distributed sequence of nonequilibrium states varying in time. During an irreversible process the system as well as parts of it ("subsystems") interact with each other. The processes of interaction are described by rate (evolution) equations. A fundamental equation together with an appropriate set of rate equations form the concept of a constitutive law favored by nonconventional thermodynamicists.

Without attemping to complete this sketchy vocabulary, I shall further point out that in conventional thermodynamics stored energy does not occur but is understood as the difference in 
the values of some thermodynamic polential (internal energy, Helmholtz free energy, etc.) between two states when one of them is normalized by an (often implicit) convention. This is due to the fact that the definition of any potential leaves an arbitrary additive constant unspecified.

\section{State}

Having indicated some of the semantic problems that members of my audience may experience, I shall proceed with this exposition using the concepts and terminology of conventional thermodynamics.

The concept of state is best explained by making a distinction between the physical space and the (Gibbsian) phase or state space. Attention is centered on irreversible processes as they, necessarily, occur in a system, treated as a continuum, throughout which the states are distributed spatially and evolve in time. Accordingly, we consider an element ("point") $\Delta V(x, t)$ of a continuum B, Fig. 1, and indicate for it a selected number of extensive variables, that is the fields of internal energy per unit mass $u(x, t)$, a set of deformation parameters $a(x, t)$, and a set of internal variables $\alpha(x, t)$.

At this point it is necessary to make a digression in order to motivate the introduction of the concept of an internal variable $\alpha$, following its clearest expression by P. W. Bridgman. In our specific case we shall describe the external deformation of $\Delta V$ by the field of linearized (small) strain per unit mass $p^{-1} \varepsilon_{i j}(\mathrm{~m} A)$, with $p=c 0$ st, fully adequate for the treatment of elastic and inelastic deformations in metals. Elements $\Delta V$ of metals which can perfcrm inelastic, irreversible processes are not homogeneous and thus capable of undergoing internal transformations, such as growth of dislocations, plastic flow, diffusive migration of atoms, slow chemical reactions, etc. Observations confirm that a thermodynamic description of the internal state (deformation) of the element $\Delta V$ can be obtained by accepting P. W. Bridgman's [8] insight:*

\footnotetext{
- I cannot restrain myself from remarking that I find it difficult to understand why so compelling an interpretation has not found immediate and universal acceptance.
} 
I believe shat in general the analysis of such systems will be furthered by the recognition of a new type of large-scale thermedynamic parameter of state, namely the parameter of state which can be measured but not controlled. . These parameters are measurable, but they are not controllable, which means that they are coupled to no external force variable which might provide the means of control. And not being coupled to a force variable, they cannot take part in mechanical work.

The view is that, connected to the outside, each site would produce work

$$
d W^{\circ}{ }_{\text {int }}=A_{j} d \alpha_{j} \quad \text { (sum for } n \text { sites) }
$$

reversibly. Terms of the type of Eqn. (1) do not appear in the field equations which govern the evolution of the fields in time.

Reverting to the physical space, Fig. 1, we note that the nonequilibrium element $\Delta V$ interacts with its surroundings during an irreversible process through a transfer of work and heat. In general, these interactions are described by the field $F(x, t)$ of generalized forces (in our case $F \equiv \sigma_{\mathrm{ij}}$ - the Cauchy stress), the work being

$$
\mathrm{d} W=\mathbf{F} \cdot \mathrm{da} \text { (or }-\rho^{-1} \sigma_{\mathrm{ij}} \mathrm{d} \varepsilon_{\mathrm{ij}} \text { in our case) }
$$

The transfer of heat is described by the heat-flux vector field $q(x, t)$. In contrast with $a$ and $\alpha, F$ and $q$ cannot be added over the volume elements $\Delta V$ to produce a value for the system as a whole.

The equilibrium state of an element is uniquely described by the set

$$
\{u, \mathbf{a}, \alpha],
$$

and the interaction vectors $\mathbf{F}$ and $\mathbf{q}$ vanish identically. Such a state can be conveniently represented as a point in a space with coordinates $u, a, \alpha$ sketched in Fig. 2, the Gibbsian state space. A curve $R$ in this space represents a reversible process and it is implied that the internal sites are now connected to the outside. Thus, the work element on curve $R$ is the sum

\footnotetext{
Work done by the system is reckoned positive, and the usual convention for stress is followed.
} 


$$
d W^{\circ}=F^{\circ} \cdot d a+A^{0} \cdot d \alpha .
$$

At this point the reader must realize that the forces, F, required to produce work on element $\Delta V$ in the physical space will be different from those which act on it during a hypothetical, reversible process $\mathrm{R}$. By contrast, the extensive variables $u, \boldsymbol{a}, \boldsymbol{\alpha}$ have been so selected that the corresponding nonequilibrium quantities possess unambiguous meanings in physical space.

In the case of a metal, Eqn. (4) is written

$$
\mathrm{d} W^{0}=-p^{-1} \sigma_{\mathrm{ij}} \mathrm{d} \varepsilon_{\mathrm{ij}}+A_{\mathrm{k}}^{0} \mathrm{~d} \alpha_{\mathrm{k}}
$$

and the Gibbsian state space consists of seven variables, $u, p^{-1} \varepsilon_{i j}$, owing to the symmetry $\varepsilon_{\mathrm{ij}}=\varepsilon_{\mathrm{ji}}$.

\section{Accompanying equilibrium state: accompanving reversible process}

Whereas the set (3) is sufficient to specify a unique equilibrium state, the number of parameters needed unambiguously to specify a nonequilibrium state is much larger, given that the set (3) is included already. This situation is illustrated in Fig. 3. The base manifold symbolizes the hyperspace of equilibrium states $r(u, 2, \alpha)$. An irreversible process I starts with an equilibrium state $e_{1}$ and develops nonequilibrium states $n$ which must reach outside of $r$. For the purpose of further analysis, we shall associate an equilibrium state $r$ with every state $n$, so that

$$
u(r)=u(n), \quad \alpha(r)=\alpha(n), \quad \alpha(r)=\alpha(n) .
$$

We shall call $r$ the acsompanying equilibrium state to nonequilibrium state $n$. The possibility of this association rests on the already mentioned observation that $u, \mathbf{a}, \alpha$ have a defined meaning for nonequilibrium as well as for equilibrium states. The same cannot be said about the conjugate, intensive quantities $F$ and $F^{\circ}$. Underlying the transformation $n \rightarrow r$ is the Gedankenexperiment illustrated in Fig. 4. We imagine that the element $\Delta V$ in state $n$ is 
suddenly surrounded by a rigid enclosure so that no external work $\mathbf{F}$ - da can be done on it and $a$ is thus kept constant. We further postulate that the enclosure must be adiabatic, inhibiting the flow of heat. Finally, we let the internal variables $\alpha$ be arrested (frozen), so that $\alpha=$ const during $n \rightarrow r$. Since no work or heat is exchanged along $n \rightarrow r$, the internal energy, $u$, must also remain constant. In this manner the conditions of this thought experiment guarantee that conditions (6) are satisfied, and we describe process $n \rightarrow r$ as one of an adiabatic projection in the enlarged state space. The process of equilibration from $n$ to $r$ will involve a characteristic relaxation time $\tau$, made nondimensional by the external characteristic time $\mathbf{a} / \mathbf{a}$ and describable by the Deborah number

$$
D e=\frac{\tau}{a / d}
$$

We can go further and regard the reversible process $R$, imbedded in the space of equilibrium states, as the accompanying reversible process to 1 . We postulate that the rate at which I progresses in time is imposed on $R$, so that we picture here a reversible process as one which occurs at a finite rate in contrast with the historic presumption that a reversible process must occur "infinitely slowly" or must be the result of the convergence from a real process when its rate is reduced to zero. I do not think that the concept of a reversible process occurring at a finite rate does violence to our physical imagination. However, if the audience members remain unpersuaded they can imagine that, departing from equilibrium at any state $r$, the system keeps continuously reverting to it" with a negligibly short relaxation time $\left(D_{e}=0\right)$.

The various proponents of unconventional versions of thermodynamics often make proposals regarding an enlargement of the manifold of states so that it would also include states of nonequilibrium in which $R$ could be imbedded. However, no single, convincing proposal has yet arisen. In this lecture we emphatically do not enlarge the Gibbsian space in order to pave the way for a clear statement of the principle of local state. The insistence on

- Say, by the coaxing of Maxwell's demon. 
choosing exclusively the set (3) of extensive variables has made it possible to define a unique accompanying equilibrium state $r$ to a nonequilibrium state $n$. We remind our audience that the conjugate intensive variables have different values at $n$ and at $r$ so that the inclusion of even one of them in the state space would make a clear adiabatic projection troublesome.

\section{Entropy and thermodynamic temperature}

Many battles have been fought over the "correct" definition of the concept of entropy (which people widely use and abuse) and of the way to assign numerical values of entropy to states. The controversial problem is with nonequilibrium states. The principle of local state bypasses this issue in an ad hoc, heuristic way. For this reason, we shall merely recall, for easy reference, how this problem is handled for equilibrium states.

Here we fall back on any standard reasoning (e.g., Caratheodory's) which asserts that the expression for heat

$$
d Q^{\circ}=d u+d W^{\circ}
$$

along a reversible process (curve $R$ in state space) possesses an integrating denominator $T(u, \mathrm{a}, \alpha)$, which can be interpreted as thermodynamic temperature (First Part of Second Law). Thus,

$$
\mathrm{d} s(u, \mathrm{a}, \alpha)=\frac{\mathrm{d} Q^{\circ}}{T}=\frac{\mathrm{d} u+F^{\circ} \cdot \mathrm{da}+\mathrm{A}^{\circ} \cdot \mathrm{d} \alpha}{T(u, \mathbf{a}, \alpha)}
$$

is a perfect differential assuring the existence of a fundamental equation

$$
s=s(u, \mathrm{a}, \alpha)
$$

which must represent a convex manifold in the state space.

Numerical values of entropy are calculated by integration in the state space,

$$
s(u, \mathbf{a}, \alpha)-s_{0}=\int_{\mathrm{R}} \frac{\mathrm{d} Q^{\circ}}{T},
$$


Where $R$ in an arbitrary curve in that space, and thus, always representis a reversible process. Here $s_{0}$ is an arbitrary constant because the integral $(10)$ has the mathematical properties of a potential. The fact that $s$ is a potential leads to additional, very important relations:

$$
\text { reciprocity relations } \frac{1}{T}=\frac{\partial s(u, a, \alpha)}{\partial u} \text {, }
$$

etc., for $F / T$ and $A / T$, and

$$
\text { reciprocal relations (Maxweil) } \frac{\partial u}{\partial a_{i}}=\frac{\partial F_{i}}{\partial u},
$$

etc., etc.

It should be noted that the integral (10) cannot be evaluated in the physical space, contrary to what is cften claimed in nonconventional versions of the subject.

\section{Byposhesis of local equilibcium \\ (Principle of local state)}

Semantically, the phrase hypothesis of local equilibrium is a misnomer, and for this reason many of us prefer to call it principle of local state. The purpose of this principle, as already stated earlier, is to assign a heuristically justified numerical value of entropy to a nonequilibrium state. Explicit use of this principle was introduced into continuum thermodynamics independently by J. Meixner [1] in 1941 and I. Prigogine [2] in 1947.

Disregarding this semantic disagreement, I quote here its statement from the book by S. R. de Groot and P. Mazur [3].

It will now be assumed that although the total system is not in equilibrium, there exists within small mass elements a state of "local equilibrium" for which the local entropy s is the same function as in real equilibrium. In particular, we arsume that the fundamental equation of state remains valid for a mass element along its center-of-mass motion. This hypothesis of "local" equilibriwn can, from a macroscopic point of 
view, onty be justified by virtue of the validity of the conclusions derived from it.

The gist of this statement has the effect of associating with a nonequilibrium state, such as that of element $\Delta V$ in Fig. 1, the entropy and thermodynamic temperature of the accompanying state. In particular, as is evident from the Gedankenexperiment pictured in Fig. 4, the entropy $s(r)$ of the accompanying equilibrium state must be larger than that of the nonequilibrium state $s(n)$ because the former is produced from the latter by an adiabatic, no work process, so that

$$
s(r)-s(n)=\sigma>0, \quad[s(r)=s(n)]
$$

(Second Part of the Second Law), and that the two temperatures are not exactly equal either,

$$
T(r) \neq T(n) \quad[T(r) \approx T(n)] .
$$

The principle of local state disregards (13) and (14) and introduces equalities instead, as indicated in the square brackets above.

Historically, the use of the principle of local state has led to furious controversies and verbal battles. The acceptance of the equalities by many thermodynamicists was made palatable by the assertion that they are valid when "the nonequilibrium state is close to equilibrium." Although frequently the equilibrium state in the above sentence is not specified and no measure of distance from equilibrium is provided, we can think of this in terms of the relaxation times $\tau$ and an appropriate Deborah number. Here the reader may consult Ref. 11.

My own conviction is a pragmatic one. The principle should be accepted in the mechanics of solids because of its proven performance in many fields, especially in fluid mechanics as emphasized earlier in Section 1. This is the only principle which leads a research worker to an explicit algorithm for the calculation of nurnerical values of entropy. No other competing version of thermodynamics has done this."

\footnotetext{
With the incipient exception of Extended Irreversible Thermodynamics.
} 


\section{Entropy balance. Entropy production}

From the practical point of view, the most important result of the local-state formalism is the establishment of an entropy-balance equation for continuum and the derivation from it of an explicit expression for the local rate of entropy production.

Depending on an author's taste, it is possible to start with a postulate for the expression for the entropy flux or to postulate the entropy-balance equation in its entirety; it is then called the Clausius-Duhem inequality. In our presentation this takes the form of an equality and a postulate requiring non-negativity of entropy production.

First, it is assumed that the entropy equation must have the standard form of a balance,

$$
\text { rate of change }+\operatorname{div}(f l u x)=\text { source }
$$

with

$$
f l u x=q / T .
$$

Equation (15) is obtained from a combination of the energy balance (First Law) writen in the physical space and the Gibbs equation written in phase space but expressed in rate form. The latter is an expression for the accompanying reversible process with the rate of the irreversible process impressed upon it in accordance with the discussion in Section 5.

The energy equation is

$$
p \frac{d u}{d t}=\sigma_{\mathrm{jj}} \frac{d \varepsilon_{\mathrm{ij}}}{\mathrm{d} t}-\frac{\partial q_{\mathrm{k}}}{\partial x_{\mathrm{k}}} .
$$

The Gibbs equation in rate form is

$$
\frac{\mathrm{d} u}{\mathrm{~d} t}=T \frac{\mathrm{d} s}{\mathrm{~d} t}+\frac{1}{\rho} \sigma_{\mathrm{ij}}^{\circ} \frac{\mathrm{d} \varepsilon_{\mathrm{ij}}}{\mathrm{dt}} \cdot A_{\mathrm{m}}^{\circ} \frac{\mathrm{d} \alpha_{\mathrm{m}}}{\mathrm{d} t} .
$$


Note the distinction between $\sigma_{\mathrm{ij}}$ in (16) and $\sigma_{\mathrm{ij}}$ in (17), often overlooked even by otherwise careful suthors. This can be traced to the fact that sometimes it is adequate to assume

$$
\sigma_{\mathrm{ij}}=\sigma_{\mathrm{ij}} \text {, }
$$

an assumption patently inadmissible in the derivation of the Navier-Stokes equations.

It is now a simple matter to calculate the Lagrangian derivative $\rho d s / d y$ by eliminating du/dt from Eqns. (16) and (17) and casting the result in the form of a standard balance after the pattern of Eqn. (15). We obtain

$$
\rho \frac{\mathrm{d} s}{\mathrm{~d} t}+\frac{\partial}{\partial x_{\mathbf{k}}} \frac{q_{\mathrm{k}}}{T}=\dot{\theta}
$$

where the local rate of entropy production is given explicitly as

$$
\hat{\theta}=q_{\mathrm{k}}\left(\frac{\partial T^{-1}}{\partial x_{\mathrm{K}}}\right)+\frac{\sigma_{\mathrm{ij}}-\sigma_{\mathrm{ij}}^{\circ}}{T} \dot{\varepsilon}_{\mathrm{ij}}+\frac{\rho A^{\circ} \mathrm{m}}{T} \dot{\alpha}_{\mathrm{m}} \geq 0
$$

The requirement that $\theta$ must be positive is a, sometimes disputed, expression of the Second part of the Second Law of thermodynamics.

It is noted that Eqn. (20) represents entropy production as a sum of products of generalized forces into generalized fluxes.

The expression in Eqn. (20) suggests consistent forms of rate equations. These must be written to satisfy the intrinsic symmetries of the system. It is often claimed that the rate equations must be linear in the forces and fluxes. I do not perceive this to be mandatory, even though in many concrete cases the relations turn out to be genuinely linear. The requirement of linearity if rooted in a reasoning which supposes that the form of $\dot{\theta}$ must be consistent with that which results from a first-order Chapman-Enskog solution of the Boltzmann equation for the distribution function of low-density monatomic gases. But even this derivation admits nonlinear force-flux relations in the presence of chemical reactions, as demonstrated by I. Prigogine [3]. 
A combination of the standard balance equations of mass, momentum and energy with the rate equations yields a closed system of partial differential equations; their solution, subject to prescribed initial and boundary conditions, constitutes the process. 


\section{References}

[1] J. Meixner and H. G. Reik, Thermotynamik der irreversiblen Prozesse, contribution to Handbuch der Physik, Vol. m/2, ed. by S. Flugge (Springer, 1959), pp. 413-523.

also

J. Meixner, Thermodynamik irreversiblen Prozesse, Aachen, 1954.

[2] I. Prigogine, Etude thermod'ynamique des phenomenes irreversibles, Liège, 1947.

also

I. Prigogine, Introduction to Thermodynamics of Irreversible Processes, Wiley, 1967.

[3] S. R. de Groot and P. Mazur, Non-Equilibrium Thermodynamics, North-Holland, 1962 and Dover, 1984.

[4] J. W. Gibbs, Lettir of acceptance of the Rumford Medal, 1881. See Josiah Willard Gibbs, The History of a Great Mind, by L. P. Wheeler, Yale University Press, 1951, p. 155.

[5] J. Kestin and J. Bataille, Thermodynamics of Solids, Proc. of 3rd Int. Symposium on Continuum Models of Discrete Systeins (Freudenstadt), ed. by E. Kröner and K. H. Anthony, U. of Waterloo Press, 1980, pp. 99-147.

[6] D. G. Miller, The Onsager Relations; Experimental Evidence, in Foundations of Continuum Thermodynamics, ed. by J. J. Delgadn Domingos, M. N. R. Nina and J. H. Whitelaw, Wiley, 1973, pp. 185-214.

[7] P. W. Bridgman, The Thermodynamics of Plastic Deformation and Generalized Entropy. Rev. Mod. Phys. 22 (1950), pp. 56-63.

[8] P. W. Bridgman, The Nature of Thermodymamics, Harvard University Press, 1941, and Harper \& Brothers, 1961.

[9] J. Kestin and J. R. Rice, Paradoxes in the Application of Thermodynamics to Strained Solids, in A Critical Review of Thermodynamics, ed. by E. B. Stuart, A. J. Brainard, and B. Gal-Or, Mono Book Corp., 1970, pp. 275-298.

[10] C. Truesdell, Rational Thermodynamics, Springer, 1984.

[11] J. Kestin, A Note on the Relation between the Hyporhesis of Local Equilibrium and the Clausius-Duhem Inequality, J. Non-Eq. Thermo. 15 (1990), pp. 193-212. 
Appendix: The local-state (local-equilibrium) formalism (consecutive steps)

1. Formulate work in neversible process

$$
d W^{0}=F^{0} \cdot d a+A^{0} \cdot d \alpha .
$$

2. Write down the Gibbs equation

$$
d u=T d s-F^{\circ} \cdot d a-A^{0} \cdot d \alpha .
$$

3. Establish fundamental equation

$$
s=s(u, a, \alpha) \text { in space }\{u, a, \alpha\}
$$

note that all variables are intunsive;

use Maxwell's relations;

postulate convexity for intrinsic stability.

3a. For $\mathrm{T}=$ const analysis use Legendre transform

$$
d(u-T s)=d f=-s d T-F^{\circ} \cdot d a-A^{0} \cdot d \alpha,
$$

and $f=f(a, \alpha)$ with $T$ as parameter

4. First Law in physical space

$$
\text { here set } a \equiv \varepsilon_{\mathrm{ij}} / p \text { and } F=\sigma_{\mathrm{ij}}
$$

$$
\rho \frac{d u}{d t}=\sigma_{i j} \frac{d \varepsilon_{i j}}{d t}-\frac{\partial q_{k}}{\partial x_{k}} ;
$$

$\varepsilon_{i j}$ satisfies compatibility 
5. Gibbs equation in rate form

$$
\frac{d u}{d t}=T \frac{d s}{d t}+\frac{1}{p} \sigma_{i j} \frac{d \varepsilon_{i j}}{d t}-A_{m}^{\circ} \frac{d \alpha_{m}}{d t}
$$

6. Eliminate du/dt from 4. and 5.

$$
\frac{d s}{\rho_{d t}}=\frac{\sigma_{i j}-\sigma_{i j}^{\circ}}{T} \varepsilon_{i j}+\frac{A_{m}^{0}}{T} \dot{\alpha}_{m}-\frac{1}{T} \frac{\partial q_{k}}{\partial x_{k}} .
$$

7. Transform 6. to standard balance equation form and use $\mathrm{q}_{\mathrm{i}} / \mathrm{T}$ as entropy flux

$$
\begin{aligned}
\rho \frac{\mathrm{d} s}{\mathrm{dT}}+\frac{\partial}{\partial x_{k}}\left(\frac{q_{k}}{T}\right)=q_{k}\left(\frac{\partial T-1}{\partial x_{k}}\right) & +\frac{\sigma_{i j}-\sigma_{i j}^{\circ}}{T} \dot{\varepsilon}_{i j}+\frac{\rho A^{0} m}{T} \dot{\alpha}_{m}=\dot{\theta}, \\
\dot{\theta} & \geq 0
\end{aligned}
$$

here $\dot{\theta}$ is the local rate of entropy production per unit volume.

8. Formulate rate equations

9. Set up field equations and solve a system of partial differential equations. 


\section{Captions to Figures}

Figure 1. Physical space

Figure 2. Gibbsian phase (state) space

Figure 3. Relation of nonequilibrium states $n$ to state space

Figure 4. Creating accompanying equilibrium state by a Gedankenexperiment 


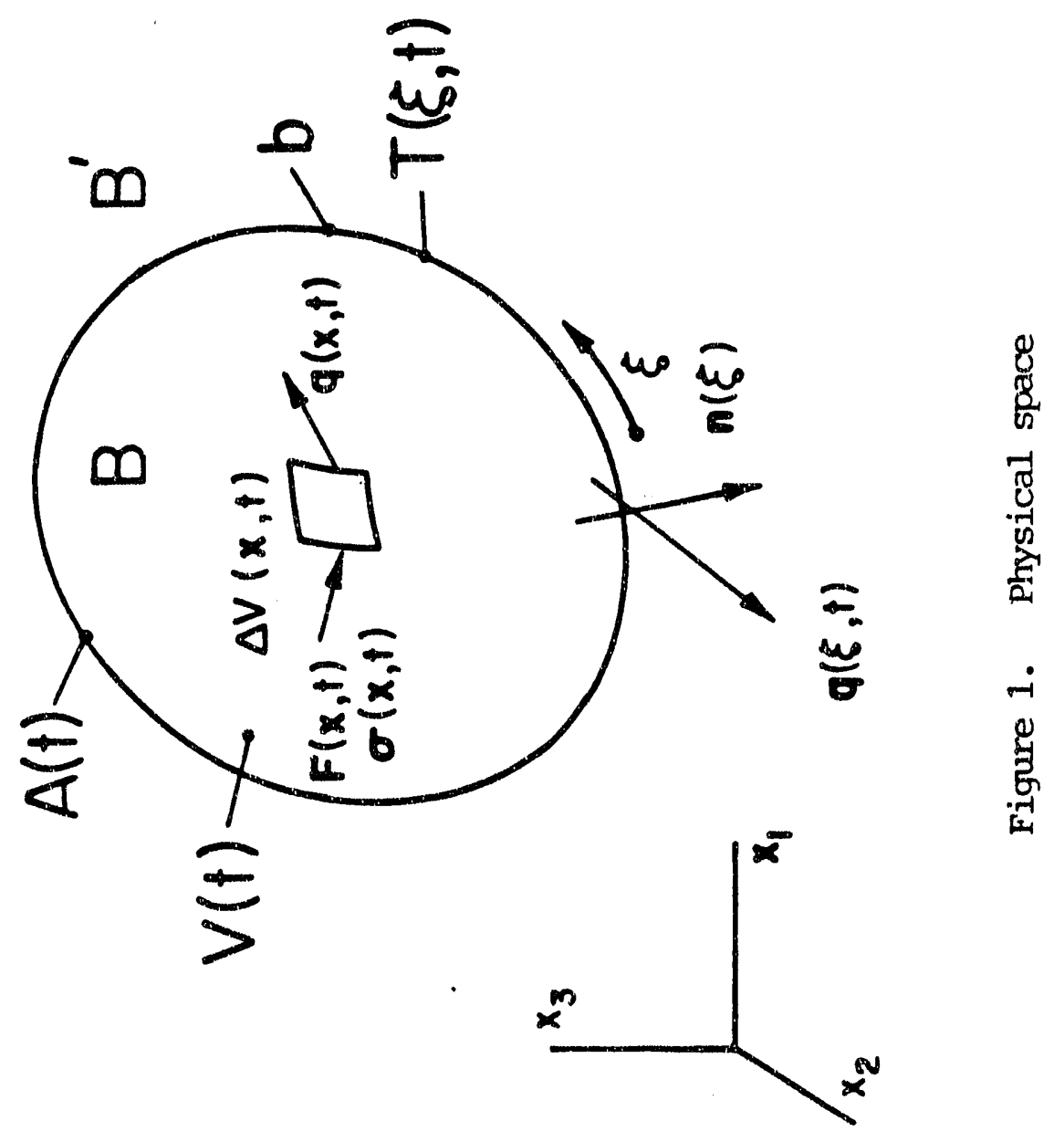




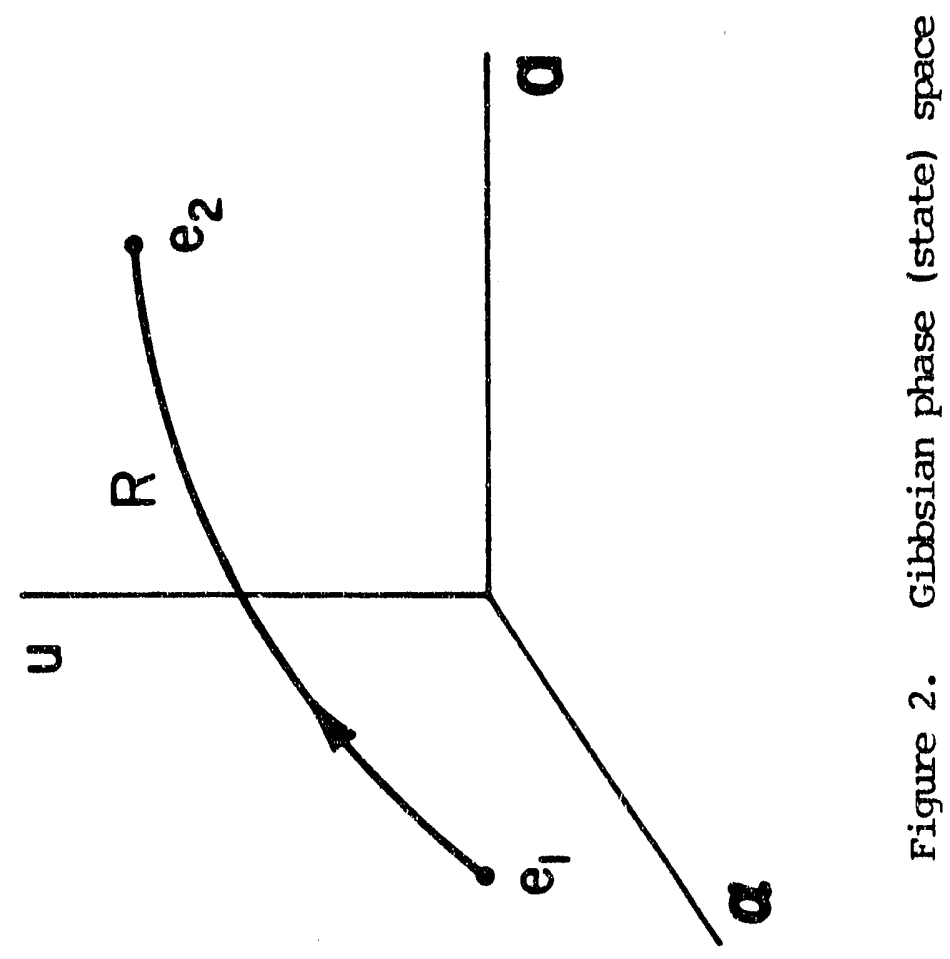




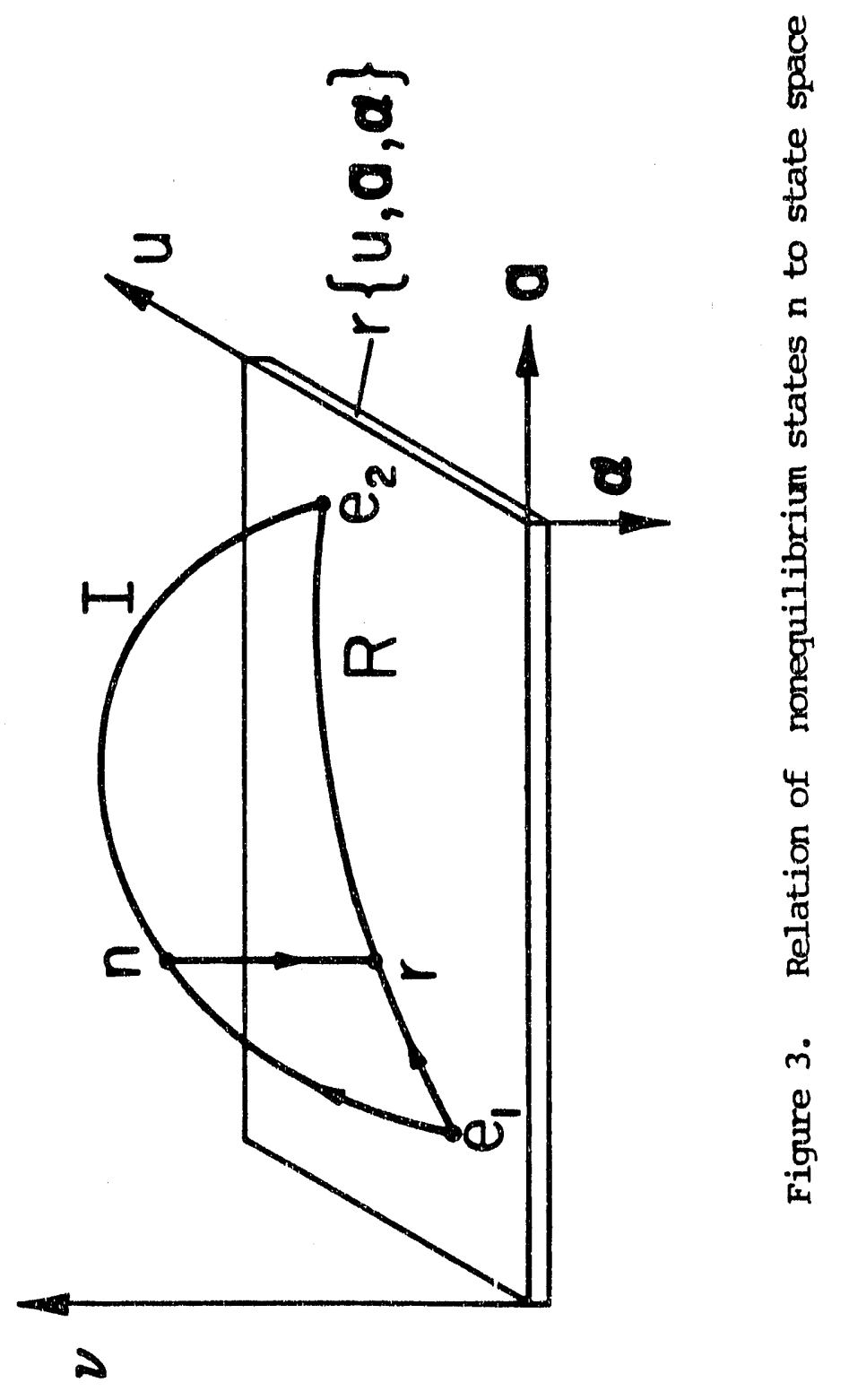




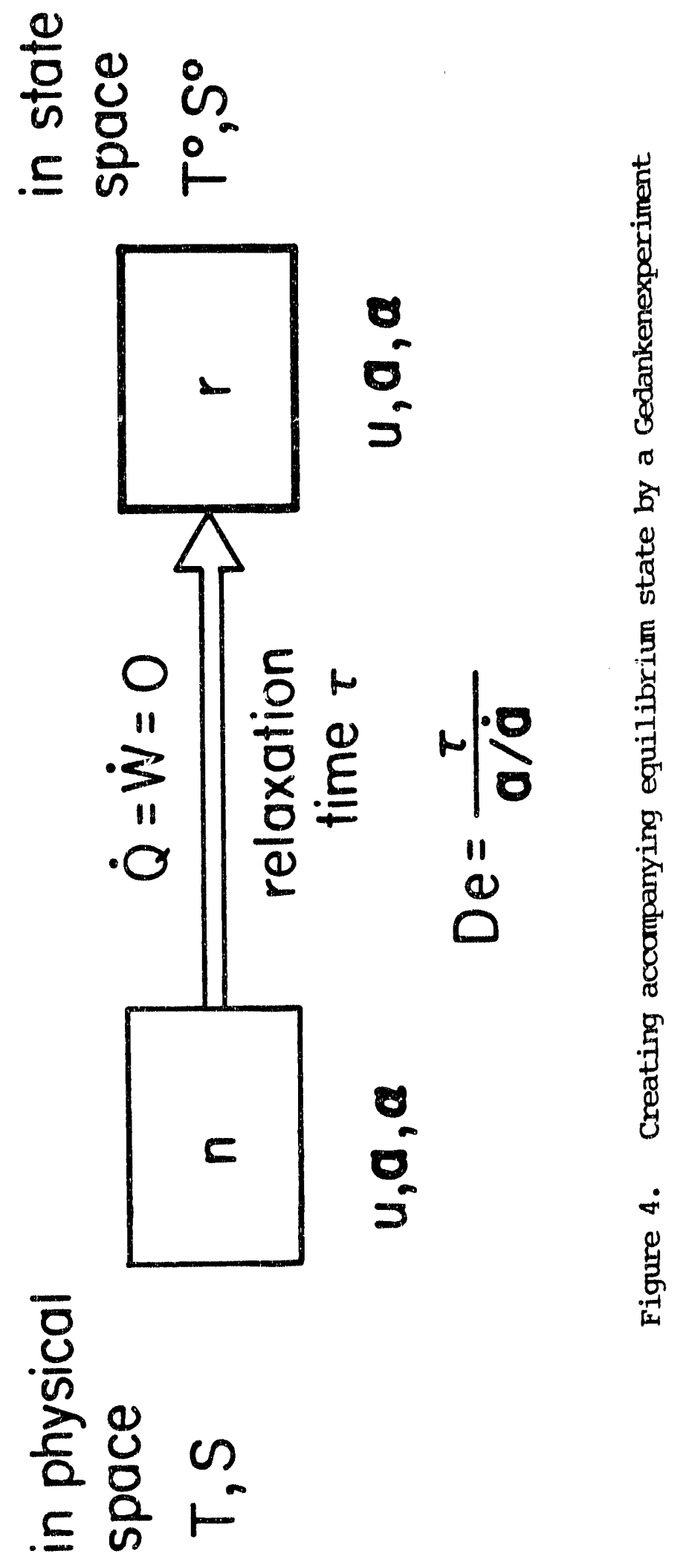



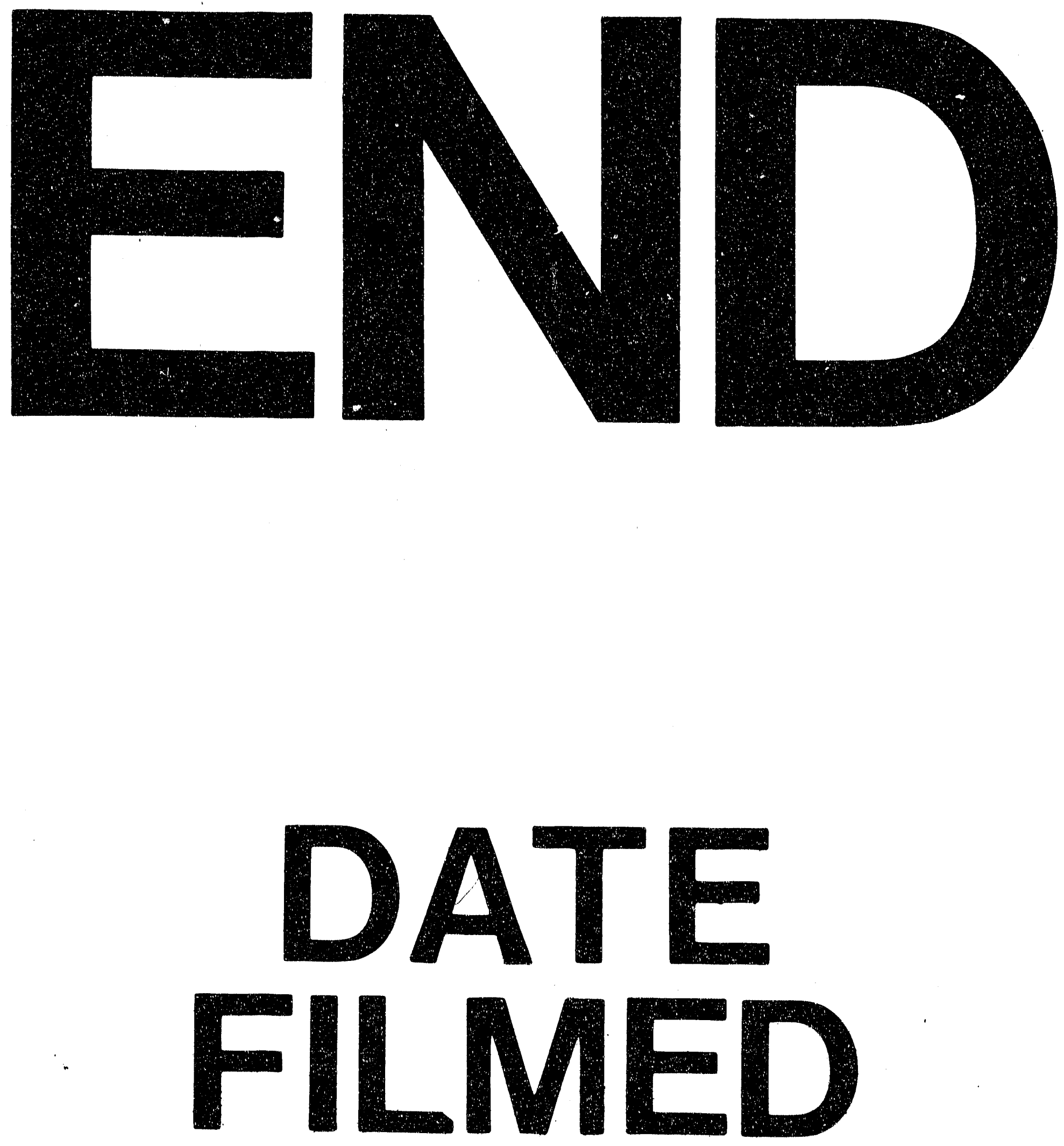

氞

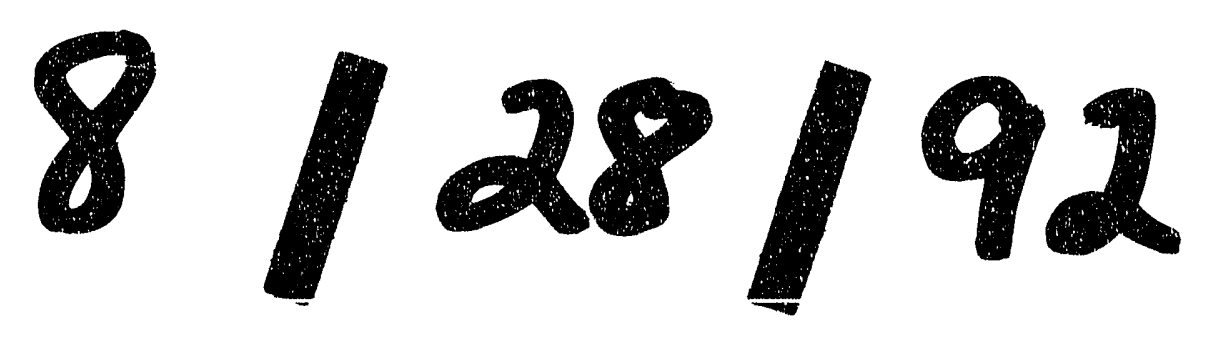

